\title{
EXPERIMENTAL MODEL OF BLUNT CHEST TRAUMA IN RATS
}

\author{
Ivan P. Novakov, \\ Delyan P. Delev', \\ Svitlana Y. Bachurska², \\ Dmitrii G. Staikov ${ }^{2}$ \\ Department of Special Surgery, \\ Medical University, Plovdiv \\ ${ }^{1}$ Department of Pharmacology, \\ Medical University, Plovdiv \\ ${ }^{2}$ Department of Pathology, Medical \\ University, Plovdiv
}

\section{Corresponding author:}

Ivan P. Novakov

e-mail: inovakov2003@yahoo.com

Received: June 30, 2014

Revision received: September 24, 2014

Accepted: November 24, 2014

\begin{abstract}
Summary
The aim of the study was to present our own experimental model of blunt chest trauma in small laboratory animals. Fifteen Wistar albino rats (body weight 290.0-320.0 g) were used in the experiment. An originally designed platform was used to inflict blunt thoracic trauma in the rats. Gross examination and histological evaluation of the heart and lung were performed. It was established that blunt chest trauma caused three thoracic injuries: bilateral lung contusion, myocardial contusion and hemopericardium. These thoracic injuries occurred as a result from direct compression of the chest wall towards the lung and the heart. The severity of lung parenchymal and myocardial pathological findings depended on the energy of blunt chest trauma. The study presents a reproducible small-animal experimental model of blunt chest trauma. The model gives opportunities for further studying different aspects of lung and myocardial contusion.
\end{abstract}

Key words: blunt chest trauma, lung contusion, myocardial contusion, rats

\section{Introduction}

Blunt chest trauma remains a significant problem in emergency medicine and trauma care. Blunt chest trauma causes a variety of injuries, ranging from simple contusion of the thoracic wall to lifethreatening injuries of the thoracic viscera. Onefourth of trauma-related deaths are specifically related to blunt chest trauma. The high morbidity and mortality of blunt chest trauma explains the great interest in its experimental exploration [1-4].

The aim of this study was to present our own experimental model of blunt chest trauma in small laboratory animals.

\section{Material and Methods}

Fifteen male Wistar albino rats (body weight 290.0$320.0 \mathrm{~g}$ ) were used. For the experimental study we used a special platform, designed by ourselves. The construction of the platform consists of several 
elements: a solid wooden base; a vertical metal rod, fixed to the base of the platform; a horizontal metal rod, mobile towards the vertical rod, an outer plastic cylinder - fixed to the horizontal rod; a trigger, fixed to an inner plastic cylinder; a falling body (Fig. 1).

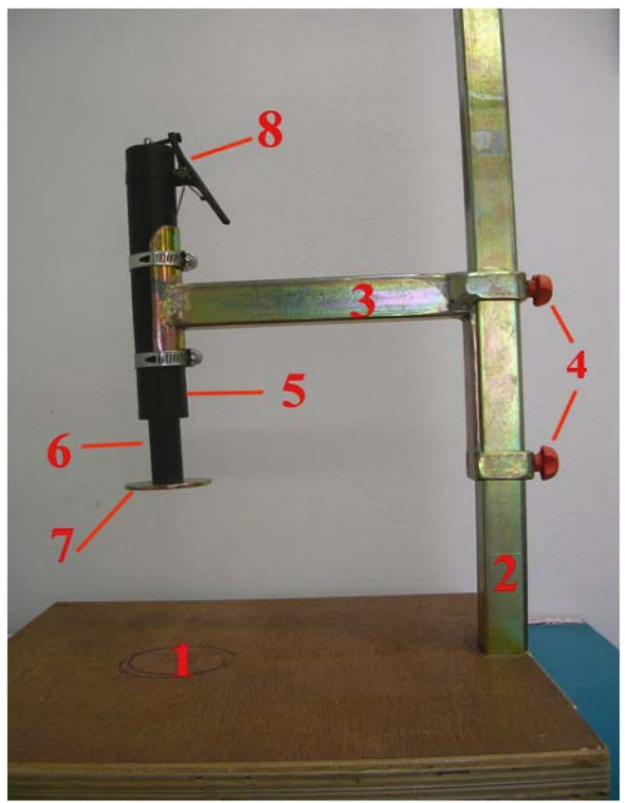

Figure 1. Platform for blunt thoracic trauma for small laboratory animals.

( 1 -wooden base; 2 - vertical metal rod; 3 -horizontal metal rod; 4 - metal bolts to fix a horizontal metal rod; 5 - outer plastic cylinder; 6 - plastic cylinder of falling body; 7 -metal plate; 8 -trigger)

The falling body of the platform consisted of a plastic cylinder, a flat round metal base, and a metal bolt that closed the upper pole of the plastic cylinder. The plastic cylinder of the falling body was filled with pellets (diameter $0.75 \mathrm{~mm} /$ caliber 13 each). The small pellets, filling the plastic cylinder allowed for changing the weight of the falling body in a range of 250 to $950 \mathrm{~g}$. We designed four metal plates of the falling body with different diameters: 40, 50, 60 and $70 \mathrm{~mm}$. The metal bolt closing the upper pole of the cylinder allowed the falling body to hang on the trigger of the platform (Fig. 1). When the trigger was pressed, the falling body was released by the horizontal rod and fell free onto the base of the platform. The construction of the platform made it possible to change the height of the horizontal rod and changing the distance between falling body and the base of the platform. The animals were placed on the base of the platform, laid on their right side, just under the falling body that hung on the trigger.

Blunt chest trauma was caused by free fall of the body upon the left thoracic wall of the animals. The kinetic energy of the mechanical impact on the thoracic cage of the animals is a function of the weight of the falling body and its height of dropping. The energy of the trauma (in joules) was calculated using the formula $E=m g h$ (J) [m - mass of the falling body (kilograms); $\mathrm{g}-$ gravitational acceleration $=9.81 \mathrm{~m} / \mathrm{s}^{2} ; \mathrm{h}-$ height that falling body is dropped from (meters)].

The animals were divided into five subgroups (three in each) on the basis of the kinetic energy of the impact on the thoracic wall: $1.8 \mathrm{~J}, 2.0 \mathrm{~J}, 2.2$ $\mathrm{J}, 2.4 \mathrm{~J}$, and $2.6 \mathrm{~J}$. The weight of the falling body was $500 \mathrm{~g}$. With that weigh of the falling body, its height of dropping towards the thoracic cage of the animals had to be $37 \mathrm{~cm}, 41 \mathrm{~cm}, 45 \mathrm{~cm}, 49 \mathrm{~cm}$ and $53 \mathrm{~cm}$ to generate mechanical impacts with the energies that mentioned above.

At the moment of blunt chest trauma the animals were in a state of general anesthesia (intraperitoneal injection of ketamine hydrochloride $90 \mathrm{mg} / \mathrm{kg}$ ). The animals were euthanized at the $24^{\text {th }}$ hour after the thoracic trauma by a lethal dose of thiopental natrium $(>100 \mathrm{mg} / \mathrm{kg})$. The animals were immediately autopsied by opening the chest. The heart and lungs were excised from the chest. Gross examination and histopathological evaluation was made in a blinded fashion by two experienced laboratory pathologists. Histological sections of the heart and lungs (stained with hematoxylin and eosin - H\&E) were examined by light microscopy under x 10, $\mathrm{x}$ 20, x 40 magnification.

This experimental study was conducted with the permission of the local ethics committee of Medical University, Plovdiv (№2/2012) and National Bulgarian Safety Food Agency (№79/2013).

\section{Results}

All fifteen rats survived to the $24^{\text {th }}$ hour after the blunt thoracic.

Gross pathology analysis established lung contusion in all the animals. There were areas of hemorrhages, as a sign of lung contusion, on the surface of both lungs (Fig. 2; Fig. 3). 


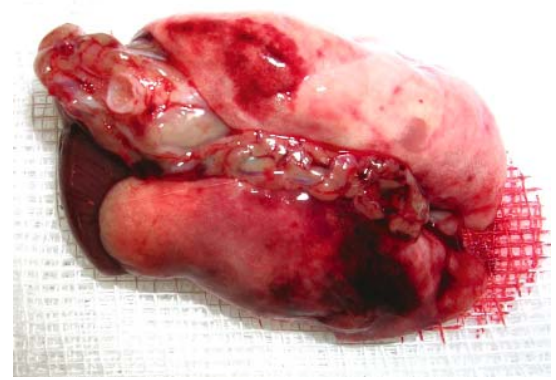

Figure 2. Lung contusion in blunt chest trauma with energy of $2.2 \mathrm{~J}$ - gross appearance. Hemorrhages on lateral surfaces of both lungs

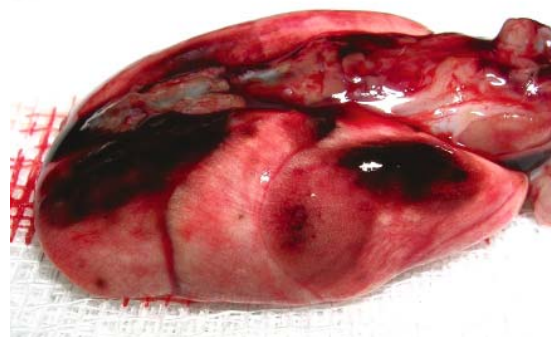

Figure 3. Lung contusion in blunt chest trauma with energy of 2.6 J-gross appearance. Severe hemorrhages on lateral surfaces of the left lung

Heart gross pathological findings were not established in the animals of the $1.8 \mathrm{~J}$ subgroup. Areas of hemorrhages, as a sign of myocardial contusion, were seen on the heart surface in one of the animals of the $2.0 \mathrm{~J}$ subgroup and in all the $2.2 \mathrm{~J}, 2.4 \mathrm{~J}$ and $2.6 \mathrm{~J}$ subgroups (Fig. 4). Hemopericardium was established in two of the animals - one of the $2.4 \mathrm{~J}$ subgroup of and another in the $2.6 \mathrm{~J}$ subgroup (Fig. 5).

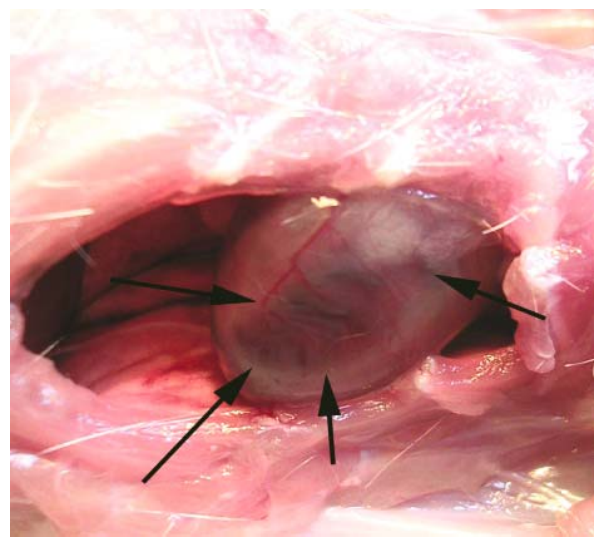

Figure 4. Myocardial contusion in blunt chest trauma with 2.6 J energy - gross appearance. Black arrows show the hemorrhages on the surface of the heart

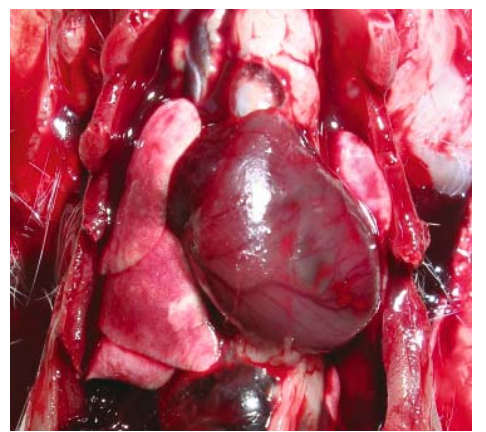

Figure 5. Blunt chest trauma with $2.6 \mathrm{~J}$ energy gross appearance. Bilateral lung contusion, hemopericardium and heart contusion are demonstrated

Histological examination established myocardial and lung contusion in all animals. Parenchymal lung hemorrhages - interstitial and alveolar, alveolar destruction and injured bronchioles were found (Fig. 6; Fig. 7).

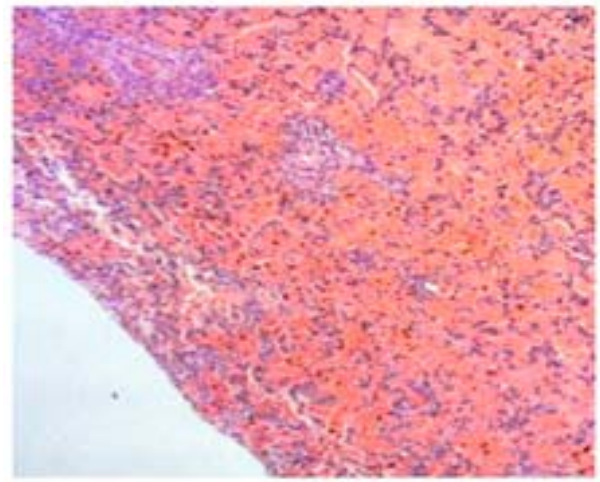

Figure 6. Severe lung parenchymal hemorrhage in blunt chest trauma with $2.6 \mathrm{~J}$ kinetic energyhistological examination (H\&Ex10)

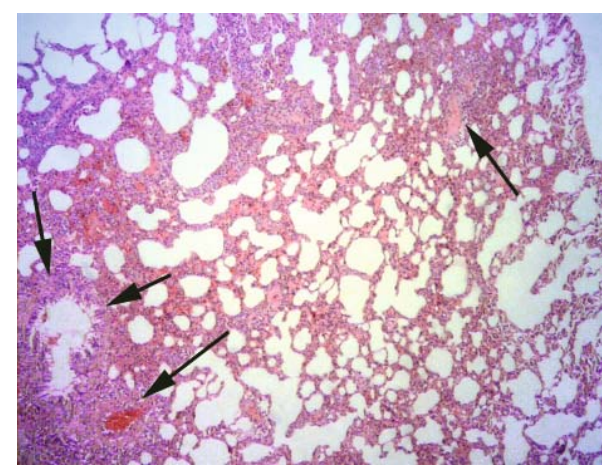

Figure 7. Alveolar destruction and injured bronchioles (marked with black arrows) in blunt chest trauma - histological examination (H\&Ex20)

Areas of myocardial and subepicardial hemorrhages were found (Fig. 8; Fig. 9). It was established that severity of histological 
myocardial and lung parenchymal pathological changes depended on the energy of the inflicted blunt chest trauma.

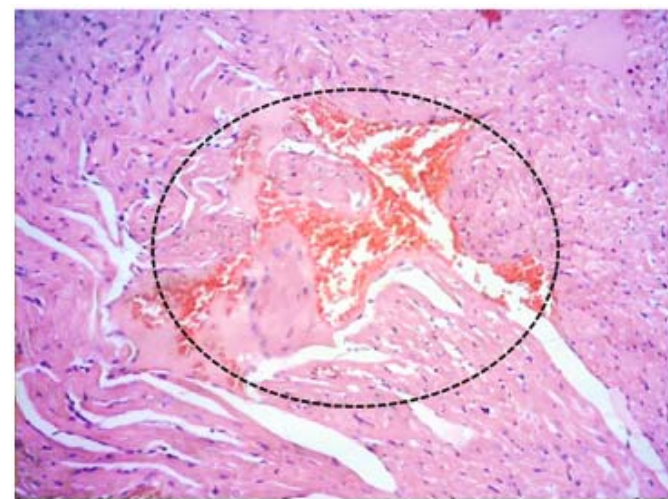

Figure 8. Myocardial hemorrhage in blunt chest trauma with energy $1.8 \mathrm{~J}$ - histological examination (H\&Ex20)

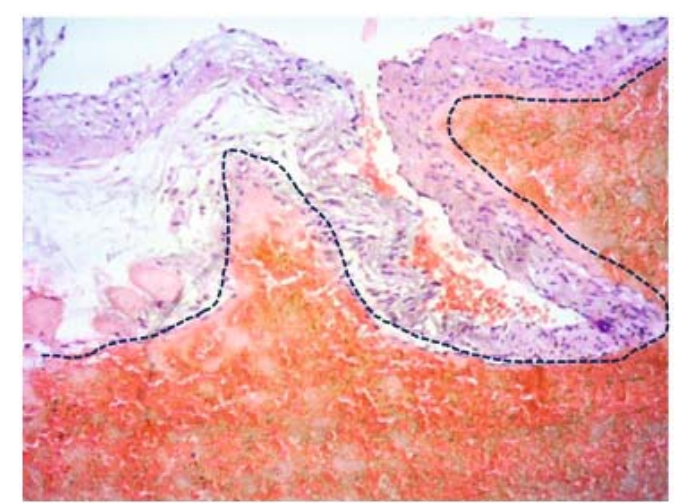

Figure 9. Severe subepicardial heart hemorrhage in blunt chest trauma with energy $2.6 \mathrm{~J}$ - histological examination (H\&Ex 10)

\section{Discussion}

Because of its high incidence and high level of mortality, blunt chest trauma is considered as a serious problem in emergency medicine and thoracic surgery practice [1-4]. These characteristics of blunt chest trauma explain the great interest in its experimental exploration [5]. For the needs of our scientific work we also needed an experimental model of blunt chest trauma, which is presented in this study.

We had to choose the species of animals for our model of blunt chest trauma. There are limitations in using large-animal models of chest trauma. Some of the reasons for such limitations are: complex technical supply, frequent mortality of the animals and high cost of the experiments [6-9]. Because of that, many authors have used models of blunt chest trauma [10-13].

We decided to use Wistar albino rats were used for our experimental model of blunt chest trauma since these rats are very prolific and highly resistant to unfavourable effects, e.g. as anesthetics and mechanical impact. Moreover, many cell- and mediator-specific reagents, which are widely available for small animals such as rats are available [5].

Nowadays, experimental blunt chest trauma is most commonly is caused by mechanical impact through dropping a solid body onto the chest wall of laboratory animals [10-13]. This mechanism was adopted by our scientific team. We designed and constructed a special platform, unique for this study. The platform is simple in construction, easy to use and allowed for precise determination of the kinetic energy of mechanical impact on the thoracic cage of the animals.

Using the results of other published experimental studies, we decided to cause blunt chest trauma by a mechanical blow on the thoracic wall of the animals with kinetic energies of $1.8 \mathrm{~J}, 2.0 \mathrm{~J}, 2.2 \mathrm{~J}, 2.4 \mathrm{~J}$ and $2.6 \mathrm{~J}$ [8-10]. Our results showed that even with relatively high energy of mechanical impact $(2.4 \mathrm{~J}, 2.6 \mathrm{~J})$ the animals survived the blunt chest trauma.

Three kinds of injuries of the thoracic viscera were caused by the mechanical impact on the left thoracic wall of the animals: lung contusion, myocardial contusion and hemopericardium. As was expected, lung contusion was the most common thoracic injury in our model of blunt chest trauma, which was also confirmed on gross examination and on histological analysis in all animals. Mechanical impact on the left thoracic wall causes left-sided pulmonary contusion. At the moment of a left-sided blow, the right lung was also under pressure as a result of pressing the right chest wall against the solid base of the platform. This explains why left-sided lateral mechanical impact on the thoracic cage caused bilateral pulmonary contusion.

Myocardial contusion was the second thoracic injury in our model of blunt chest trauma. The injury of the heart was a result of sudden compression of the chest wall at the moment of blunt chest trauma. In contrast to the tender lung, the heart is steadier to mechanical impacts. It explains why the gross pathological lung findings were more obvious than those of the heart.

Lung and myocardial contusions are considered as potentially life-threatening injuries 
that should be detected during a secondary assessment of blunt chest trauma patients [4]. As was expected, histological analysis established that myocardial and lung pathological changes correlated with the energy of the blunt chest trauma.

In contrast to lung and myocardial contusions, cardiac tamponade is one of the immediate lifethreatening thoracic injuries that require evaluation and treatment during the primary survey. Cardiac tamponade is caused by the accumulation of blood in the pericardial sac hemopericardium [4]. We established hemopericardium in two of the animals. The amount of blood in the pericardial sac was not sufficient to compress the hearts of the animals. Because of that, the animals survived up to the $24^{\text {th }}$ hour after the blunt chest trauma.

\section{Conclusions}

We present a reproducible small-animal experimental model of blunt chest trauma that causes three deadly thoracic injuries: lung contusion, myocardial contusion and hemopericardium.

The presenting model of blunt chest trauma provides opportunities for further studying various aspects of lung and myocardial contusion. Furthermore, the construction of the platform makes it possible to cause experimental blunt trauma to in anatomical regions other than the chest.

\section{References}

1. Wanek S. Blunt thoracic trauma: flail chest, pulmonary contusion and blast injury. Crit Care Clin. 2004;20(1):71-81.

2. Demetriades D, Murray J, Charalambides K, Alo $\mathrm{K}$, Velmahos $\mathrm{G}$, Rhee P, et al. Trauma fatalities: time and location of hospital deaths. J Am Coll Surg. 2004;198(1):20-6.
3. Martin SK, Martin SK, Shatney CH, Sherck JP, Ho CC, Homan SJ, Neff J, et al. Blunt trauma patients with prehospital pulseless electrical activity (PEA): poor ending assured. J Trauma. 2002;53(5):876-80; discussion 880-1.

4. Yamamoto L, Schroeder C, Beliveau C. Thoracic trauma. The deadly dozen. Crit Care Nurs. 2005; 28:22-40.

5. Cohn SM, DuBose JP. Pulmonary contusion: an update on recent advances in clinical management. World J Surg. 2010;34(8):1959-70.

6. Hellinger A, Konerding $M$, Malkusch W, Obertacke U, Redl H, Bruch J, et al. Does lung contusion affect both the traumatized and the noninjured lung parenchyma? A morphological and morphometric study in the pig. J Trauma. 1995;39(4):712-9.

7. Moseley RV, Vernick JJ, Doty DB. Response to blunt chest injury: a new experimental model. J Trauma. 1970;10(8):673-83.

8. Doty DB, Anderson AE, Rose EF, Go RT, Chiu CL, Ehrenhaft. Cardiac trauma: clinical and experimental correlation of myocardial contusion. Ann Surg. 1974;180(4):452-60.

9. Fulton RL, Peter ET. The progressive nature of pulmonary contusion. Surgery. 1970;67(3):499506.

10. Özel SK, Özel HB, Colakoglu NM, Ilhan N, Arslan N, Ozan E. Protective effect of the thoracic cage on parenchyma in response to trauma in blunt thoracic trauma: an experimental study. Ulus Travma Acil Cerrahi Derg. 2010;16 (4):287-92.

11. Raghavendran K, Davidson BA, Helinski EH, Marschke CJ, Manderscheid P, Woytash JA, et al. $A$ rat model for isolated bilateral lung contusion from blunt chest trauma. Anesth Analg. 2005;101(5):1482-9.

12. Raghavendran K, Davidson BA, Woytash JA, Helinski JD, Marschke CJ, Manderscheid PA, et al. The evolution of isolated bilateral lung contusion from blunt chest trauma in rats: cellular and cytokine responses. Shock. 2005;24(2):132-8.

13. Wang ND, Stevens MH, Doty DB, Hammond EH. Blunt chest trauma: an experimental model for heart and lung contusion. J Trauma. 2003;5494):744-8. 